\title{
SMALL ESSENTIAL SPECTRAL RADIUS PERTURBATIONS OF OPERATORS WITH TOPOLOGICAL UNIFORM DESCENT
}

\author{
QINGPING ZENG ${ }^{\bowtie}$, HUAIJIE ZHONG and ZHENYING WU
}

(Received 16 April 2011)

\begin{abstract}
In this paper we consider small essential spectral radius perturbations of operators with topological uniform descent-small essential spectral radius perturbations which cover compact, quasinilpotent and Riesz perturbations.
\end{abstract}

2010 Mathematics subject classification: primary 47A53; secondary 47A55.

Keywords and phrases: Banach spaces, operator ranges, topological uniform descent, essential spectral radius, perturbation.

\section{Introduction}

In [9] Grabiner studied the structure and perturbation theory of operators with topological uniform descent. This class of operators contains the semi-Fredholm operators, most of the generalizations of Fredholm operators which appear in the literature, and various classes of operators defined by means of kernels and ranges (see $[2-6,16,19])$. For a bounded linear operator $T$ with topological uniform descent, Grabiner's study focuses on the sequences of ranges, $\left\{R\left(T^{n}\right)\right\}$, and of kernels, $\left\{N\left(T^{n}\right)\right\}$, and on the analogous sequences for sufficiently small or compact perturbations of $T$. The present paper is concerned with small essential spectral radius perturbations (that is, the essential spectral radius of the perturbation is small) of operators with topological uniform descent-small essential spectral radius perturbations which cover compact, quasinilpotent and Riesz perturbations. As seen in Remark 4.5, our perturbational results extend all compact perturbational results of Grabiner [9], and some sufficiently small perturbational results of Grabiner [9]. Most of our perturbational results seem to be new even for Fredholm operators.

Throughout this paper, $\mathcal{B}(X)$ (respectively, $\mathcal{K}(X)$ ) will denote the set of all bounded (respectively, compact) linear operators on an infinite-dimensional complex Banach

This work has been supported by the Specialized Research Fund for the Doctoral Program of Higher Education (2010350311001) and the Natural Science Foundation of Fujian Province (NO. 2009J01005).

(C) 2011 Australian Mathematical Publishing Association Inc. 0004-9727/2011 \$16.00 
space $X$. For an operator $T \in \mathcal{B}(X)$, let $T^{*}$ denote its dual, $N(T)$ its kernel, $\alpha(T)$ its nullity, $R(T)$ its range and $\beta(T)$ its defect. Recall that the descent and the ascent of $T \in \mathcal{B}(X)$ are given by $\operatorname{dsc}(T)=\inf \left\{n \in \mathbb{N}: R\left(T^{n}\right)=R\left(T^{n+1}\right)\right\}$ and $\operatorname{asc}(T)=\inf \{n \in \mathbb{N}:$ $N\left(T^{n}\right)=N\left(T^{n+1}\right)$ \}, respectively. If the range $R(T)$ of $T \in \mathcal{B}(X)$ is closed and $\alpha(T)<\infty$ (respectively, $\beta(T)<\infty$ ), then $T$ is said to be upper semi-Fredholm (respectively, lower semi-Fredholm). If $T \in \mathcal{B}(X)$ is both upper and lower semi-Fredholm, then $T$ is said to be Fredholm. If $T \in \mathcal{B}(X)$ is either upper or lower semi-Fredholm, then $T$ is said to be semi-Fredholm, and its index is defined by $\operatorname{ind}(T)=\alpha(T)-\beta(T)$. We call $T \in \mathcal{B}(X)$ upper semi-Browder (respectively, lower semi-Browder) if $T$ is upper semiFredholm (respectively, lower semi-Fredholm) and has finite ascent (respectively, descent). Also, the hyperrange and hyperkernel of $T \in \mathcal{B}(X)$ are the subspaces of $X$ defined by $R\left(T^{\infty}\right)=\bigcap_{n=1}^{\infty} R\left(T^{n}\right)$ and $N\left(T^{\infty}\right)=\bigcup_{n=1}^{\infty} N\left(T^{n}\right)$, respectively. Recall that $T \in \mathcal{B}(X)$ is called bounded below if $T$ is injective and has closed range $R(T)$. We call $T \in \mathcal{B}(X)$ semi-regular if $R(T)$ is closed and $N(T) \subseteq R\left(T^{\infty}\right)$. Further, $T \in \mathcal{B}(X)$ is called essentially semi-regular if $R(T)$ is closed and there exists a finite-dimensional subspace $F \subseteq X$ such that $N(T) \subseteq R\left(T^{\infty}\right)+F$.

If $T \in \mathcal{B}(X)$, for each nonnegative integer $n, T$ induces a linear transformation from the vector space $R\left(T^{n}\right) / R\left(T^{n+1}\right)$ to the space $R\left(T^{n+1}\right) / R\left(T^{n+2}\right)$. We will let $k_{n}(T)$ be the dimension of the null space of the induced map and let $k(T)=\sum_{n=0}^{\infty} k_{n}(T)$. Further, denote $k\left(T^{\infty}\right)=\sup _{n \in \mathbb{N}} k\left(T^{n}\right)$. It follows easily from [9, Theorem 3.7] that $T \in \mathcal{B}(X)$ is essentially semi-regular if and only if $R(T)$ is closed and $k(T)$ is finite. Moreover, from [9, Lemma 3.11], we know that $k(T)$ is finite if and only if $k\left(T^{\infty}\right)$ is finite, and that $k\left(T^{\infty}\right)$ is the dimension of $\left(R\left(T^{\infty}\right)+N\left(T^{\infty}\right)\right) / R\left(T^{\infty}\right)$. We also set $c_{n}(T)=$ $\operatorname{dim} R\left(T^{n}\right) / R\left(T^{n+1}\right)$ and $c_{n}^{\prime}(T)=\operatorname{dim} N\left(T^{n+1}\right) / N\left(T^{n}\right)$. Recall that the essential descent and the essential ascent of $T \in \mathcal{B}(X)$ are given by $\operatorname{dsc}_{e}(T)=\inf \left\{n \in \mathbb{N}: c_{n}(T)<\infty\right\}$ and $\operatorname{asc}_{e}(T)=\inf \left\{n \in \mathbb{N}: c_{n}^{\prime}(T)<\infty\right\}$, respectively. The following definition, which was introduced by Grabiner in [9], describes the classes of operators we will study in this paper.

Definition 1.1. Let $T \in \mathcal{B}(X)$ and let $d \in \mathbb{N}$. Then $T$ has uniform descent for $n \geq d$ if $k_{n}(T)=0$ for all $n \geq d$. If, in addition, $R\left(T^{n}\right)$ is closed in the operator range topology of $R\left(T^{d}\right)$ for all $n \geq d$, then we say that $T$ has topological uniform descent, and, more precisely, that $T$ has topological uniform descent for $n \geq d$.

The Calkin algebra over a Banach space $X$ is the quotient algebra $C(X)=$ $\mathcal{B}(X) / \mathcal{K}(X)$, and $\pi: \mathcal{B}(X) \rightarrow C(X)$ denotes the natural homomorphism. Let $r_{e}(T)$ denote the spectral radius of the element $\pi(T)$ in $C(X), T \in \mathcal{B}(X)$, that is, $r_{e}(T)=$ $\lim _{n \rightarrow \infty}\left\|\pi\left(T^{n}\right)\right\|^{1 / n}$; this is called the essential spectral radius of $T$. An operator $T \in \mathcal{B}(X)$ is Riesz if $\{\lambda \in \mathbb{C}: T-\lambda I$ is not Fredholm $\}=\{0\}$, that is $r_{e}(T)=0$.

For $T \in \mathcal{B}(X)$, the reduced minimum modulus, the injection modulus and the surjection modulus of $T$ are defined by $\gamma(T)=\inf _{x \notin N(T)}(\|T x\| / \operatorname{dist}(x, N(T)))($ if $T=0$ then we set $\gamma(T)=\infty), m(T)=\inf \{\|T x\|: x \in X,\|x\|=1\}$ and $q(T)=\sup \{\varepsilon \geq 0: T U \supseteq$ $\varepsilon U\}$, respectively. Note that $\gamma(T)=\gamma\left(T^{*}\right)$ for every $T \in \mathcal{B}(X) ; \gamma(T)>0$ if and 
only if $R(T)$ is closed [1, Theorem 1.13]; the injection modulus $m(T)$ (respectively, surjection modulus $q(T)$ ) of an operator $T$ which is bounded below (respectively, onto) is a special case of the reduced minimum modulus $\gamma(T)$. For a detailed discussion of the injection modulus and the surjection modulus of $T$, we refer the reader to [17, pp. 26-28].

If $T \in \mathcal{B}(X)$ is semi-Fredholm, then there exists $\varepsilon>0$ such that both $\alpha(T-\lambda I)$ and $\beta(T-\lambda I)$ are constant for all $0<|\lambda|<\varepsilon$ (one of the two constants may be infinite). In [20], Zemánek has defined the stability radius $\delta(T)$ to be the supremum of all $\varepsilon>0$ with the property described above. That is,

$$
\delta(T)=\sup \{\varepsilon>0: \alpha(T-\lambda I) \text { and } \beta(T-\lambda I) \text { are constant for all } 0<|\lambda|<\varepsilon\} .
$$

For $T \in \mathcal{B}(X)$, let us define the injectivity radius, the surjectivity radius, and the semi-regular radius of $T$ as follows respectively:

$$
\begin{aligned}
s_{\text {inj }}(T) & =\sup \{\varepsilon \geq 0: T-\lambda I \text { is bounded below for all }|\lambda|<\varepsilon\} ; \\
s_{\text {sur }}(T) & =\sup \{\varepsilon \geq 0: T-\lambda I \text { is onto for all }|\lambda|<\varepsilon\} ; \\
s_{\mathrm{se}}(T) & =\sup \{\varepsilon>0: T-\lambda I \text { is semi-regular for all }|\lambda|<\varepsilon\} .
\end{aligned}
$$

The semi-Fredholm radii of $T$ are

$$
\begin{aligned}
& r_{+}(T)=\sup \{\varepsilon \geq 0: T-\lambda I \text { is upper semi-Fredholm for all }|\lambda|<\varepsilon\} ; \\
& r_{-}(T)=\sup \{\varepsilon \geq 0: T-\lambda I \text { is lower semi-Fredholm for all }|\lambda|<\varepsilon\} .
\end{aligned}
$$

We remark that $r_{+}(T) \geq s_{\text {inj }}(T)$ and $r_{-}(T) \geq s_{\text {sur }}(T)$.

This paper is organized as follows. In Section 2 we gather together some preliminary results which we will need repeatedly later. Section 3 is devoted to the study of small essential spectral radius perturbations of operators with topological uniform descent. Our study proceeds in two steps. Suppose that $T$ has topological uniform descent for $n \geq d$. First, using some technical lemmas, we research, in Theorem 3.8, small essential spectral radius perturbations of the restriction $\left.T\right|_{R\left(T^{d}\right)}$, or (what amounts to the same thing) small essential spectral radius commuting perturbations of semi-regular operators. Second, we consider small essential spectral radius perturbations of operators with topological uniform descent. The final perturbational results are obtained by determining properties of the perturbed operators from properties of their restrictions to $R\left(T^{d}\right)$. In Section 4 we give some comments on small essential spectral radius perturbations. Specifically, we show, as we stated at the beginning of this paper, that small essential spectral radius perturbations cover compact, quasinilpotent and Riesz perturbations (see Remark 4.5).

Henceforth, for a subspace $M$ of a Banach space $X, \bar{M}$ means the closure of $M$ in $X$. If $M$ is an invariant subspace of an operator $T \in \mathcal{B}(X)$, the symbols $\left.T\right|_{M}$ and $T_{M}$ mean the restriction of $T$ to $M$ and the map induced by $T$ on $X / M$. 


\section{Preliminary results}

In this section we gather together some preliminary results which we will need repeatedly later. The following propositions can be found in [9, Lemmas 2.2, 3.6 and 4.5, Theorem 3.4], [18, Theorem 1] and [14, Lemma 15], respectively.

Proposition 2.1. Suppose that $T$ is a bounded linear operator with closed range from the Banach space $X$ to the Banach space $Y$. If $E$ and $F$ are linear subspaces of $X$ and $Y$, respectively, and if $E \supseteq N(T)$ and $F \subseteq R(T)$, then:

(a) $T(\bar{E})=\overline{T(E)}$;

(b) $T^{-1}(\bar{F})=\overline{T^{-1}(F)}$.

Proposition 2.2. If $T \in \mathcal{B}(X)$ has topological uniform descent for $n \geq d$, then:

(a) the restriction of $T$ to $R\left(T^{\infty}\right)$ is onto;

(b) the map induced by $T$ on $R\left(T^{d}\right) / R\left(T^{\infty}\right)$ is bounded below;

(c) the restriction of $T$ to $R\left(T^{d}\right) \cap \overline{N\left(T^{\infty}\right)}$ is onto;

(d) the map induced by $T$ on $X / \overline{N\left(T^{\infty}\right)}$ is bounded below.

Proposition 2.3. If $T \in \mathcal{B}(X)$ has topological uniform descent for $n \geq d$, then:

(a) $R\left(T^{\infty}\right)+N\left(T^{d}\right)=R\left(T^{\infty}\right)+N\left(T^{\infty}\right)=R\left(T^{\infty}\right)+\overline{N\left(T^{\infty}\right)}$;

(b) $R\left(T^{d}\right) \cap N\left(T^{\infty}\right)=R\left(T^{\infty}\right) \cap N\left(T^{\infty}\right)$;

(c) $R\left(T^{d}\right) \cap \overline{N\left(T^{\infty}\right)}=R\left(T^{\infty}\right) \cap \overline{N\left(T^{\infty}\right)}$;

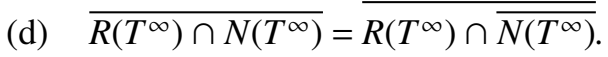

Proposition 2.4. If $T$ and $V$ are commuting linear transformations on the vector space $X$, and $n$ and $d$ are nonnegative integers, then:

(a) the map induced by $T^{d}$ from $R\left(V^{n}\right) / R\left(V^{n+1}\right)$ to $R\left(V^{n} T^{d}\right) / R\left(V^{n+1} T^{d}\right)$ is onto and has kernel naturally isomorphic to $\left(R\left(V^{n}\right) \cap N\left(T^{d}\right)\right) /\left(R\left(V^{n+1}\right) \cap N\left(T^{d}\right)\right)$;

(b) the map induced by the identity on $X$ from $\left(N\left(V^{n+1}\right) \cap R\left(T^{d}\right)\right) /\left(N\left(V^{n}\right) \cap R\left(T^{d}\right)\right)$ to $N\left(V^{n+1}\right) / N\left(V^{n}\right)$ is one-to-one and has cokernel naturally isomorphic to $\left(N\left(V^{n+1}\right)+R\left(T^{d}\right)\right) /\left(N\left(V^{n}\right)+R\left(T^{d}\right)\right)$;

(c) $T^{d}$ induces an isomorphism from $N\left(T^{d+n+1}\right) / N\left(T^{d+n}\right)$ onto $\left(N\left(T^{n+1}\right) \cap\right.$ $\left.R\left(T^{d}\right)\right) /\left(N\left(T^{n}\right) \cap R\left(T^{d}\right)\right)$.

Proposition 2.5. Suppose that $T$ and $S$ are commuting bounded operators on the Banach space $X$, and that $V=S+T$. Then:

(a) if $T$ is an upper semi-Browder operator and $r_{e}(S)<r_{+}(T)$, then $V$ is an upper semi-Browder operator;

(b) if $T$ is a lower semi-Browder operator and $r_{e}(S)<r_{-}(T)$, then $V$ is a lower semiBrowder operator.

Proposition 2.6. Let $T \in \mathcal{B}(X)$ and let $M$ be its closed invariant subspace. Then $r_{e}\left(\left.T\right|_{M}\right) \leq r_{e}(T)$ and $r_{e}\left(T_{M}\right) \leq r_{e}(T)$.

Next, we recall some classical results. 
Makai and Zemánek proved [15, Theorems 1,3] the following elegant formulas on the asymptotic behavior of $m$ and $q$ :

$$
\begin{aligned}
& s_{\text {inj }}(T)=\lim _{n \rightarrow \infty} m\left(T^{n}\right)^{1 / n}, \\
& s_{\text {sur }}(T)=\lim _{n \rightarrow \infty} q\left(T^{n}\right)^{1 / n},
\end{aligned}
$$

for any $T \in \mathcal{B}(X)$. Zemánek proved [20, Theorem 1] the following elegant formula:

$$
\delta(T)=\lim _{n \rightarrow \infty} \gamma\left(T^{n}\right)^{1 / n},
$$

for any semi-Fredholm operator $T$. Kordula and Müller proved [13, Theorems 3, 4] that similar formulas hold for semi-regular operators and essentially semi-regular operators. Specifically, if $T$ is semi-regular then

$$
s_{\mathrm{se}}(T)=\lim _{n \rightarrow \infty} \gamma\left(T^{n}\right)^{1 / n},
$$

while if $T$ is essentially semi-regular then

$$
\sup \{\varepsilon>0: T-\lambda I \text { is semi-regular for all } 0<|\lambda|<\varepsilon\}=\lim _{n \rightarrow \infty} \gamma\left(T^{n}\right)^{1 / n} .
$$

By (2.1) and (2.2), we know that if $T$ is bounded below then

$$
s_{\text {inj }}(T)=\lim _{n \rightarrow \infty} \gamma\left(T^{n}\right)^{1 / n},
$$

while if $T$ is onto then

$$
s_{\text {sur }}(T)=\lim _{n \rightarrow \infty} \gamma\left(T^{n}\right)^{1 / n} .
$$

For the latest discussions of various operator quantities, we refer the reader to [21].

\section{Small essential spectral radius perturbations of operators with topological uniform descent}

Let $T \in \mathcal{B}(X)$. For every $d \in \mathbb{N}$, to study the topological properties of the maps induced by $T$, we will always assume that $R\left(T^{d}\right)$ is given the 'unique' operator range topology under which it becomes a Banach space continuously imbedded in $X$. Using the isomorphism $X / N\left(T^{d}\right) \approx R\left(T^{d}\right)$ and following [9], we define a topology on $R\left(T^{d}\right)$ as follows.

Definition 3.1. Let $T \in \mathcal{B}(X)$. For every $d \in \mathbb{N}$, the operator range topology on $R\left(T^{d}\right)$ is defined by the norm $\|\cdot\|_{R\left(T^{d}\right)}$ such that for all $y \in R\left(T^{d}\right)$,

$$
\|y\|_{R\left(T^{d}\right)}=\inf \left\{\|x\|: x \in X, y=T^{d} x\right\} .
$$

For a detailed discussion of operator ranges and their topologies, we refer the reader to [7, 8]. For convenience, when we refer to the operator range topology on $R\left(T^{d}\right)$, we always mean the topology described above. If $S, T \in \mathcal{B}(X)$ and $S T=T S$, then the norm of the restriction $\left.S\right|_{R(T)}:\left(R(T),\|\cdot\|_{R(T)}\right) \rightarrow\left(R(T),\|\cdot\|_{R(T)}\right)$ will be denoted by $\left\|\left.S\right|_{R(T)}\right\|_{R(T)}$. The next result shows that $\left\|\left.S\right|_{R(T)}\right\|_{R(T)}$ is no greater than $\|S\|$. 
Proposition 3.2. If $S, T \in \mathcal{B}(X), S T=T S$, then for every $n \in \mathbb{N}$, the restriction $\left.S^{n}\right|_{R(T)}$ : $\left(R(T),\|\cdot\|_{R(T)}\right) \rightarrow\left(R(T),\|\cdot\|_{R(T)}\right)$ is bounded, and $\left\|\left.S^{n}\right|_{R(T)}\right\|_{R(T)} \leq\left\|S^{n}\right\|$.

Proof. For arbitrary $\varepsilon>0$ and $y \in R(T)$ such that $\|y\|_{R(T)}=1$, there exists $x \in X$ satisfying $\|x\|=1+\varepsilon$ and $y=T x$. Hence $S^{n} y=S^{n} T x=T S^{n} x$, and then

$$
\left\|S^{n} y\right\|_{R(T)}=\inf \left\{\left\|x_{0}\right\|: S^{n} y=T x_{0}\right\} \leq\left\|S^{n} x\right\| \leq\left\|S^{n}\right\| \cdot\|x\| \leq\left\|S^{n}\right\| \cdot(1+\varepsilon) .
$$

From the arbitrariness of $y \in R(T)\left(\|y\|_{R(T)}=1\right)$ and of $\varepsilon>0$, we get $\left\|\left.S^{n}\right|_{R(T)}\right\|_{R(T)} \leq$ $\left\|S^{n}\right\|$.

From Definition 1.1 we see easily that $T \in \mathcal{B}(X)$ is semi-regular if and only if $T$ has topological uniform descent for $n \geq 0$. Suppose that $T \in \mathcal{B}(X)$ has topological uniform descent for $n \geq d$. By Proposition 3.2, we know that the restriction $\left.T\right|_{R\left(T^{d}\right)}$ : $\left(R\left(T^{d}\right),\|\cdot\|_{R\left(T^{d}\right)}\right) \rightarrow\left(R\left(T^{d}\right),\|\cdot\|_{R\left(T^{d}\right)}\right)$ of $T$ to $R\left(T^{d}\right)$ is bounded. Moreover, it is easy to see that the restriction $\left.T\right|_{R\left(T^{d}\right)}$ is semi-regular, and by Proposition 2.2, $\left.T\right|_{R\left(T^{\infty}\right)}$ : $\left(R\left(T^{\infty}\right),\|\cdot\|_{R\left(T^{d}\right)}\right) \rightarrow\left(R\left(T^{\infty}\right),\|\cdot\|_{R\left(T^{d}\right)}\right)$ is onto and $T_{\overline{N\left(T^{\infty}\right)}}$ is bounded below. Since $T_{d}:=\left.T\right|_{R\left(T^{d}\right)}$ is semi-regular, by Proposition 2.2 again, the map

$$
\left.\left(T_{d}\right)\right|_{R\left(\left(T_{d}\right)^{\infty}\right)}:\left(R\left(\left(T_{d}\right)^{\infty}\right),\|\cdot\|_{R\left(T^{d}\right)}\right) \rightarrow\left(R\left(\left(T_{d}\right)^{\infty}\right),\|\cdot\|_{R\left(T^{d}\right)}\right)
$$

is onto, and the map

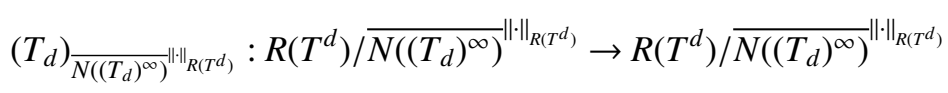

is bounded below.

From the above observation, we introduce the following notation for operators which have topological uniform descent for $n \geq d$. This notation will be used repeatedly later in this paper.

Definition 3.3. Suppose that $T \in \mathcal{B}(X)$ has topological uniform descent for $n \geq d$. Then we will denote $\left.T\right|_{R\left(T^{d}\right)},\left.T\right|_{R\left(T^{\infty}\right)}$ and $T_{\overline{N\left(T^{\infty}\right)}}$ by $T_{d}, \widehat{T}$ and $\widetilde{T}$, respectively.

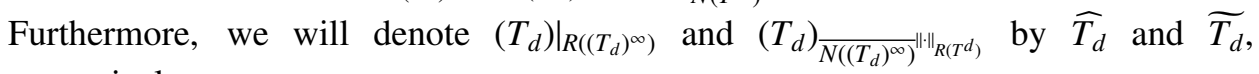
respectively.

Just as in Grabiner's study of sufficiently small or compact perturbations of operators with topological uniform descent (see [9]), we start with the special case where $T$ is semi-regular. We treat this case by applying results on perturbations of operators which are bounded below or onto to various maps induced by $T$. We start with the following lemma (see Proposition 2.5) concerning small essential spectral radius perturbations of operators which are bounded below or onto.

Lemma 3.4. Suppose that $T$ and $S$ are commuting bounded operators on the Banach space $X$, and that $V=S+T$. Then:

(a) if $T$ is bounded below and $r_{e}(S)<r_{+}(T)$, then $V$ is upper semi-Browder, $\operatorname{ind}(V)=$ $\operatorname{ind}(T)$, and $T\left(N\left(V^{k}\right)\right)=N\left(V^{k}\right)$ for all $k \in \mathbb{N}$;

(b) if $T$ is onto and $r_{e}(S)<r_{-}(T)$, then $V$ is lower semi-Browder, $\operatorname{ind}(V)=\operatorname{ind}(T)$, and $T^{-1}\left(R\left(V^{k}\right)\right)=R\left(V^{k}\right)$ for all $k \in \mathbb{N}$. 
Proof. (a) For each $0 \leq \lambda \leq 1$, let $T_{\lambda}=T+\lambda S$. It follows from Proposition 2.5 that $T_{\lambda}$ is upper semi-Browder, for every $0 \leq \lambda \leq 1$. In particular, $V=S+T$ is upper semiBrowder. From the continuity of the index and the connectedness of the interval $[0,1]$ we then infer that $\operatorname{ind}(V)=\operatorname{ind}(T+S)=\operatorname{ind}(T)$. For all $k \in \mathbb{N}$, since $V$ is upper semiBrowder, $V^{k}$ is upper semi-Browder, and hence $N\left(V^{k}\right)$ is finite-dimensional. From the commutativity of $T$ and $S$, we know that $N\left(V^{k}\right)$ is an invariant subspace of $T$. Since $T$ is one-to-one, $\left.T\right|_{N\left(V^{k}\right)}$ is also one-to-one, and hence $T\left(N\left(V^{k}\right)\right)=N\left(V^{k}\right)$.

(b) For each $0 \leq \lambda \leq 1$, let $T_{\lambda}=T+\lambda S$. It follows from Proposition 2.5 that $T_{\lambda}$ is lower semi-Browder, for every $0 \leq \lambda \leq 1$. In particular, $V=S+T$ is lower semiBrowder. From the continuity of the index and the connectedness of the interval $[0,1]$ we then infer that $\operatorname{ind}(V)=\operatorname{ind}(T+S)=\operatorname{ind}(T)$. For all $k \in \mathbb{N}$, since $V$ is lower semiBrowder, $V^{k}$ is lower semi-Browder, and hence $R\left(V^{k}\right)$ has finite codimension in $X$. From the commutativity of $T$ and $S$, we know that $R\left(V^{k}\right)$ is an invariant subspace of $T$. Since $T$ is onto, $T_{R\left(V^{k}\right)}: X / R\left(V^{k}\right) \rightarrow X / R\left(V^{k}\right)$ induced by $T$ is onto, and hence $T^{-1}\left(R\left(V^{k}\right)\right)=R\left(V^{k}\right)$.

By Lemma 3.4, we have the following result.

Lemma 3.5. Suppose that $T \in \mathcal{B}(X)$ is semi-regular. If $S \in \mathcal{B}(X), S T=T S$ and $V=$ $S+T$, then:

(a) if $r_{e}(S)<r_{-}(\widehat{T})$, then there exists an integer $p$ such that the subspace $M=$ $V^{p}\left(R\left(T^{\infty}\right)\right)$ is closed in $X$,

$$
M=V(M)=T^{-1}(M)=T(M),
$$

and $M$ has finite codimension in $R\left(T^{\infty}\right)$;

(b) if $r_{e}(S)<r_{+}(\widetilde{T})$, then there exists an integer $q$ such that $\overline{N\left(T^{\infty}\right)}$ has finite codimension in the closed subspace $L=V^{-q}\left(\overline{N\left(T^{\infty}\right)}\right)$ and

$$
L=V^{-1}(L)=T(L)=T^{-1}(L)
$$

(c) if $r_{e}(S)<r_{-}(\widehat{T})$, then

$$
\operatorname{dim}\left(R\left(T^{\infty}\right)+R\left(V^{\infty}\right)\right) / R\left(V^{\infty}\right)<\infty
$$

if $r_{e}(S)<r_{+}(\widetilde{T})$, then

$$
\operatorname{dim}\left(\overline{N\left(T^{\infty}\right)}+\overline{N\left(V^{\infty}\right)}\right) / \overline{N\left(T^{\infty}\right)}<\infty
$$

and if $r_{e}(S)<\min \left(r_{-}(\widehat{T}), r_{+}(\widetilde{T})\right)$, then, for all $n \in \mathbb{N}$,

$$
\operatorname{dim}\left(N\left(V^{n}\right)+R(V)\right) / R(V)=\operatorname{dim} N(V) /\left(N(V) \cap R\left(V^{n}\right)\right)<\infty .
$$

Proof. (a) Since $T$ is semi-regular, from Proposition 2.2 we know that $\widehat{T}=\left.T\right|_{R\left(T^{\infty}\right)}$ is onto. By Proposition 2.6 and Lemma 3.4(b), it follows that $\widehat{V}:=\left.V\right|_{R\left(T^{\infty}\right)}$ is lower semiBrowder, and that $\widehat{T}^{-1}\left(R\left(\widehat{V}^{k}\right)\right)=R\left(\widehat{V}^{k}\right)$ for all $k \in \mathbb{N}$. Hence there exists an integer $p$ 
such that the subspace $M:=\widehat{V}^{p}\left(R\left(T^{\infty}\right)\right)=V^{p}\left(R\left(T^{\infty}\right)\right)$ satisfies $V(M)=\widehat{V}(M)=M=$ $\widehat{T}^{-1}(M)=T^{-1}(M)=T(M)$. The fourth equality above can be inferred from the argument below.

The inclusion $\widehat{T}^{-1}(M) \subseteq T^{-1}(M)$ is clear. To prove the opposite inclusion it is enough to show that for every $y \in T^{-1}(M)$, then $y \in R\left(T^{\infty}\right)$. Assume that $y \in T^{-1}(M)$. Then $T y \in M=V^{p}\left(R\left(T^{\infty}\right)\right) \subseteq R\left(T^{\infty}\right)$ and, since $T$ is semi-regular, then $y \in R\left(T^{\infty}\right)$ (see [1, Theorems 1.23 and 1.24]).

Since $\widehat{V}$ is lower semi-Browder, $\widehat{V}^{p}$ is lower semi-Browder, and hence $M=$ $\widehat{V}^{p}\left(R\left(T^{\infty}\right)\right)$ has finite codimension in $R\left(T^{\infty}\right)$. Then $M$ is closed in $R\left(T^{\infty}\right)$ so, since $R\left(T^{\infty}\right)$ is closed in $X$, we can infer that $M$ is closed in $X$.

(b) Since $T$ is semi-regular, from Proposition 2.2 we know that $\widetilde{T}=T_{\overline{N\left(T^{\infty}\right)}}$ : $X / \overline{N\left(T^{\infty}\right)} \rightarrow X / \overline{N\left(T^{\infty}\right)}$ is bounded below. By Proposition 2.6 and Lemma 3.4(a), it follows that $\widetilde{V}:=V_{\overline{N\left(T^{\infty}\right)}}: X / \overline{N\left(T^{\infty}\right)} \rightarrow X / \overline{N\left(T^{\infty}\right)}$ is upper semi-Browder, and that $\widetilde{T}\left(N\left(\widetilde{V}^{k}\right)\right)=N\left(\widetilde{V}^{k}\right)$ for all $k \in \mathbb{N}$. Hence there exists an integer $q$ such that the subspace $L^{\prime}:=\widetilde{V}^{-q}\left(\overline{N\left(T^{\infty}\right)}\right)=V^{-q}\left(\overline{N\left(T^{\infty}\right)}\right) / \overline{N\left(T^{\infty}\right)}$ satisfies $\widetilde{V}^{-1}\left(L^{\prime}\right)=L^{\prime}=\widetilde{T}\left(L^{\prime}\right)$. Let $L=V^{-q}\left(\overline{N\left(T^{\infty}\right)}\right)$. Thus $V^{-1}(L) / \overline{N\left(T^{\infty}\right)}=L / \overline{N\left(T^{\infty}\right)}=\left(T(L)+\overline{N\left(T^{\infty}\right)}\right) / \overline{N\left(T^{\infty}\right)}$. Since $T$ is semi-regular, then $\overline{N\left(T^{\infty}\right)} \subseteq R(T)$, and hence, for each $y \in \overline{N\left(T^{\infty}\right)}$, there exists $x \in X$ such that $y=T x$, so $x \in T^{-1}\left(\overline{\left.N\left(T^{\infty}\right)\right)}\right.$. From Proposition 2.2 we can obtain that $T^{-1}\left(\overline{\left.N\left(T^{\infty}\right)\right)}=\overline{N\left(T^{\infty}\right)}\right.$, and then $\overline{N\left(T^{\infty}\right)} \subseteq T\left(\overline{N\left(T^{\infty}\right)}\right) \subseteq T(L)$, hence $L=V^{-1}(L)=$ $T(L)$. Since $T^{-1}\left(\overline{\left.N\left(T^{\infty}\right)\right)}=\overline{N\left(T^{\infty}\right)}\right.$, then $L=T^{-1}(L)$. Since $\widetilde{V}$ is upper semi-Browder, $\widetilde{V}^{q}$ is upper semi-Browder, thus the subspace $\widetilde{V}^{-q}\left(\overline{N\left(T^{\infty}\right)}\right)=V^{-q}\left(\overline{N\left(T^{\infty}\right)}\right) / \overline{N\left(T^{\infty}\right)}$ is finite-dimensional, that is, $\overline{N\left(T^{\infty}\right)}$ has finite codimension in $L=V^{-q}\left(\overline{N\left(T^{\infty}\right)}\right)$.

(c) If $r_{e}(S)<r_{-}(\widehat{T})$, by (a), we know that there exists a closed subspace $M$ of $X$ such that $M=V(M)=T^{-1}(M)=T(M)$. Then we have

$$
M \subseteq R\left(V^{\infty}\right) \cap R\left(T^{\infty}\right) \subseteq R(V) \cap R\left(T^{\infty}\right) \subseteq R\left(T^{\infty}\right),
$$

and, for all $n \in \mathbb{N}$, we can get

$$
N(V) \cap \overline{N\left(T^{\infty}\right)} \subseteq N(V) \cap R\left(V^{n}\right) \subseteq N(V) .
$$

From (a) we know that $M$ has finite codimension in $R\left(T^{\infty}\right)$. Hence by (3.3) we have

$$
\operatorname{dim}\left(R\left(T^{\infty}\right)+R\left(V^{\infty}\right)\right) / R\left(V^{\infty}\right)=\operatorname{dim} R\left(T^{\infty}\right) /\left(R\left(V^{\infty}\right) \cap R\left(T^{\infty}\right)\right)<\infty .
$$

If $r_{e}(S)<r_{+}(\widetilde{T})$, by (b), we know that there exists a closed subspace $L$ of $X$ such that $L=V^{-1}(L)=T(L)=T^{-1}(L)$. Then we have

$$
\overline{N\left(T^{\infty}\right)} \subseteq \overline{N\left(T^{\infty}\right)}+N(V) \subseteq \overline{N\left(T^{\infty}\right)}+\overline{N\left(V^{\infty}\right)} \subseteq L,
$$

and, for all $n \in \mathbb{N}$, we can get

$$
R(V) \subseteq N\left(V^{n}\right)+R(V) \subseteq R\left(T^{\infty}\right)+R(V) .
$$


From (b) we know that $\overline{N\left(T^{\infty}\right)}$ has finite codimension in $L$. Hence by (3.5) we have

$$
\operatorname{dim}\left(\overline{N\left(T^{\infty}\right)}+\overline{N\left(V^{\infty}\right)}\right) / \overline{N\left(T^{\infty}\right)}<\infty .
$$

If $r_{e}(S)<\min \left(r_{-}(\widehat{T}), r_{+}(\widetilde{T})\right)$, then by (3.4) and (3.6), it is easy to see that for all $n \in \mathbb{N}$,

$$
\begin{aligned}
\operatorname{dim}\left(N\left(V^{n}\right)+R(V)\right) / R(V) & \leq \operatorname{dim}\left(R\left(T^{\infty}\right)+R(V)\right) / R(V) \\
& =\operatorname{dim} R\left(T^{\infty}\right) / R\left(T^{\infty}\right) \cap R(V)<\infty, \\
\operatorname{dim} N(V) /\left(N(V) \cap R\left(V^{n}\right)\right) & \leq \operatorname{dim} N(V) /\left(N(V) \cap \overline{N\left(T^{\infty}\right)}\right) \\
& =\operatorname{dim}\left(N(V)+\overline{N\left(T^{\infty}\right)}\right) / \overline{N\left(T^{\infty}\right)}<\infty .
\end{aligned}
$$

And from [12, Lemmas 3.5 and 2.2], we have the equality

$$
\operatorname{dim}\left(N\left(V^{n}\right)+R(V)\right) / R(V)=\operatorname{dim} N(V) /\left(N(V) \cap R\left(V^{n}\right)\right) .
$$

From the proof of the above lemma, we get the following result.

Lemma 3.6. Suppose that $T \in \mathcal{B}(X)$ has topological uniform descent for $n \geq d$. If $S \in \mathcal{B}(X), S T=T S$ and $V=S+T$, then:

(a) if $r_{e}(S)<r_{-}(\widehat{T})$, then there exists an integer $p$ such that the subspace $M=$ $V^{p}\left(R\left(T^{\infty}\right)\right)$ is closed in the operator range topology on $R\left(T^{d}\right), T(M)=M=$ $V(M) \subseteq T^{-1}(M)$, and $M$ has finite codimension in $R\left(T^{\infty}\right)$;

(b) if $r_{e}(S)<r_{+}(\widetilde{T})$, then there exists an integer $q$ such that the closed subspace $L=V^{-q}\left(\overline{N\left(T^{\infty}\right)}\right)$ satisfies $L=V^{-1}(L)=T(L)+\overline{N\left(T^{\infty}\right)}=T^{-1}(L)$ and $\overline{N\left(T^{\infty}\right)}$ has finite codimension in $L$;

(c) if $r_{e}(S)<r_{-}(\widehat{T})$, then

$$
\operatorname{dim}\left(R\left(T^{\infty}\right)+R\left(V^{\infty}\right)\right) / R\left(V^{\infty}\right)<\infty,
$$

and if $r_{e}(S)<r_{+}(\widetilde{T})$, then

$$
\operatorname{dim}\left(\overline{N\left(T^{\infty}\right)}+\overline{N\left(V^{\infty}\right)}\right) / \overline{N\left(T^{\infty}\right)}<\infty
$$

For a subspace $A$ of a Banach space $X$, let $A^{\perp}$ denote the annihilator of $A$. For any Banach spaces $X$ and $Y$, let $\mathcal{B}(X, Y)$ denote the set of all bounded linear operators from $X$ to $Y$. From the definition of the reduced minimum modulus, it is easy to verify that if $J \in \mathcal{B}(X, Y)$ is isometric and invertible and $T \in \mathcal{B}(X)$, then $\gamma(T)=\gamma\left(J T J^{-1}\right)$.

Lemma 3.7. Suppose that $T \in \mathcal{B}(X)$ is semi-regular. For $M$ in (3.1) and $L$ in (3.2), the operator $T$ induces $\left.T\right|_{M}, T_{M},\left.T\right|_{L}$ and $T_{L}$. Then:

(a) $\quad s_{\text {inj }}(\widetilde{T}) \leq s_{\text {inj }}\left(T_{M}\right), s_{\text {inj }}(\widetilde{T}) \leq s_{\text {inj }}\left(T_{L}\right)$;

(b) $\quad s_{\text {sur }}(\widehat{T}) \leq s_{\text {sur }}\left(\left.T\right|_{L}\right), s_{\text {sur }}(\widehat{T}) \leq s_{\text {sur }}\left(\left.T\right|_{M}\right)$.

Proof. Here, we need only prove the first inequality of (a) (respectively, (b)). The proof of the second inequality of (a) (respectively, (b)) is similar, and will be omitted. 
(a) Since both $\widetilde{T}=T_{\overline{N\left(T^{\infty}\right)}}$ and $T_{M}$ are bounded below, then it follows from (2.3) that

$$
\begin{gathered}
s_{\text {inj }}(\widetilde{T})=\lim _{n \rightarrow \infty} \gamma\left(\widetilde{T}^{n}\right)^{1 / n}, \\
s_{\text {inj }}\left(T_{M}\right)=\lim _{n \rightarrow \infty} \gamma\left(T_{M}^{n}\right)^{1 / n} .
\end{gathered}
$$

Hence, in order to show that $s_{\text {inj }}(\widetilde{T}) \leq s_{\text {inj }}\left(T_{M}\right)$, it is enough to show that

$$
\lim _{n \rightarrow \infty} \gamma\left(\widetilde{T}^{n}\right)^{1 / n} \leq \lim _{n \rightarrow \infty} \gamma\left(T_{M}^{n}\right)^{1 / n} .
$$

We now do this. For all $n \in \mathbb{N}$,

$$
\begin{gathered}
\gamma\left(T_{M}^{n}\right)=\gamma\left(\left(T_{M}^{n}\right)^{*}\right)=\gamma\left(\left.J\left(T^{n}\right)^{*}\right|_{M^{\perp}} J^{-1}\right)=\gamma\left(\left.\left(T^{n}\right)^{*}\right|_{M^{\perp}}\right), \\
\gamma\left(\widetilde{T}^{n}\right)=\gamma\left(\left(\widetilde{T}^{n}\right)^{*}\right)=\gamma\left(\left.Q\left(T^{n}\right)^{*}\right|_{\overline{N\left(T^{\infty}\right)}} Q^{-1}\right)=\gamma\left(\left.\left(T^{n}\right)^{*}\right|_{\overline{N\left(T^{\infty}\right)}}\right),
\end{gathered}
$$

where $J: M^{\perp} \longrightarrow(X / M)^{*}$ and $Q:{\overline{N\left(T^{\infty}\right)}}^{\perp} \longrightarrow\left(X / \overline{N\left(T^{\infty}\right)}\right)^{*}$ are isometric and invertible. By the definition of the reduced minimum modulus,

$$
\begin{aligned}
& \gamma\left(\left.\left(T^{n}\right)^{*}\right|_{M^{\perp}}\right)=\inf _{f \in M^{\perp}, f \notin N\left(\left(T^{n}\right)^{*}\right) \cap M^{\perp}} \frac{\left\|\left(T^{n}\right)^{*} f\right\|}{\operatorname{dist}\left(f, N\left(\left(T^{n}\right)^{*}\right) \cap M^{\perp}\right)}, \\
& \gamma\left(\left.\left(T^{n}\right)^{*}\right|_{N^{\perp}}\right)=\inf _{f \in N^{\perp}, f \notin N\left(\left(T^{n}\right)^{*}\right) \cap N^{\perp}} \frac{\left\|\left(T^{n}\right)^{*} f\right\|}{\operatorname{dist}\left(f, N\left(\left(T^{n}\right)^{*}\right) \cap N^{\perp}\right)},
\end{aligned}
$$

where $N=\overline{N\left(T^{\infty}\right)}$. By (3.1), $M \subseteq R\left(T^{n}\right)$, hence $N\left(\left(T^{n}\right)^{*}\right)=R\left(T^{n}\right)^{\perp} \subseteq M^{\perp}$. Since $T$ is semi-regular, then $\overline{N\left(T^{\infty}\right)} \subseteq R\left(T^{n}\right)$, and hence $N\left(\left(T^{n}\right)^{*}\right)=R\left(T^{n}\right)^{\perp} \subseteq \overline{N\left(T^{\infty}\right)}{ }^{\perp}$. Consequently,

$$
N\left(\left(T^{n}\right)^{*}\right) \cap M^{\perp}=N\left(\left(T^{n}\right)^{*}\right)=N\left(\left(T^{n}\right)^{*}\right) \cap{\overline{N\left(T^{\infty}\right)}}^{\perp} .
$$

By (3.1) again, $\overline{N\left(T^{\infty}\right)} \subseteq M$, hence

$$
M^{\perp} \subseteq \overline{N\left(T^{\infty}\right)}{ }^{\perp}
$$

From (3.7)-(3.10) we get $\gamma\left(\left.\left(T^{n}\right)^{*}\right|_{N\left(T^{\infty}\right)}\right) \leq \gamma\left(\left.\left(T^{n}\right)^{*}\right|_{M^{\perp}}\right)$ for all $n \in \mathbb{N}$, then $\gamma\left(\widetilde{T}^{n}\right) \leq$ $\gamma\left(T_{M}^{n}\right)$ for all $n \in \mathbb{N}$, and hence $\lim _{n \rightarrow \infty} \gamma\left(\widetilde{T}^{n}\right)^{1 / n} \leq \lim _{n \rightarrow \infty} \gamma\left(T_{M}^{n}\right)^{1 / n}$.

(b) Since both $\widehat{T}=\left.T\right|_{R\left(T^{\infty}\right)}$ and $\left.T\right|_{L}$ are onto, it follows from (2.4) that

$$
\begin{gathered}
s_{\text {sur }}(\widehat{T})=\lim _{n \rightarrow \infty} \gamma\left(\widehat{T}^{n}\right)^{1 / n}, \\
s_{\text {sur }}\left(\left.T\right|_{L}\right)=\lim _{n \rightarrow \infty} \gamma\left(\left.T^{n}\right|_{L}\right)^{1 / n} .
\end{gathered}
$$

Hence, in order to show that $s_{\text {sur }}(\widehat{T}) \leq s_{\text {sur }}\left(\left.T\right|_{L}\right)$, it is enough to show that

$$
\lim _{n \rightarrow \infty} \gamma\left(\widehat{T}^{n}\right)^{1 / n} \leq \lim _{n \rightarrow \infty} \gamma\left(\left.T^{n}\right|_{L}\right)^{1 / n}
$$


We now do this. For all $n \in \mathbb{N}$, by the definition of the reduced minimum modulus,

$$
\begin{gathered}
\gamma\left(\left.T^{n}\right|_{L}\right)=\inf _{x \in L, x \notin N\left(T^{n}\right) \cap L} \frac{\left\|T^{n} x\right\|}{\operatorname{dist}\left(x, N\left(T^{n}\right) \cap L\right)}, \\
\gamma\left(\left.T^{n}\right|_{R\left(T^{\infty}\right)}\right)=\inf _{x \in R\left(T^{\infty}\right), x \notin N\left(T^{n}\right) \cap R\left(T^{\infty}\right)} \frac{\left\|T^{n} x\right\|}{\operatorname{dist}\left(x, N\left(T^{n}\right) \cap R\left(T^{\infty}\right)\right)} .
\end{gathered}
$$

By (3.2), $N\left(T^{n}\right) \subseteq L$. Since $T$ is semi-regular, $N\left(T^{n}\right) \subseteq R\left(T^{\infty}\right)$. Consequently,

$$
N\left(T^{n}\right) \cap L=N\left(T^{n}\right)=N\left(T^{n}\right) \cap R\left(T^{\infty}\right) .
$$

By (3.2) again,

$$
L \subseteq R\left(T^{\infty}\right)
$$

From (3.11)-(3.14) we get $\gamma\left(\widehat{T}^{n}\right) \leq \gamma\left(\left.T^{n}\right|_{L}\right)$ for all $n \in \mathbb{N}$, and hence $\lim _{n \rightarrow \infty} \gamma\left(\widehat{T}^{n}\right)^{1 / n} \leq$ $\lim _{n \rightarrow \infty} \gamma\left(\left.T^{n}\right|_{L}\right)^{1 / n}$.

We recall that $r_{+}(T) \geq s_{\text {inj }}(T)$ and $r_{-}(T) \geq s_{\text {sur }}(T)$ for any $T \in \mathcal{B}(X)$; and that $V \in$ $\mathcal{B}(X)$ is essentially semi-regular if and only if $R(V)$ is closed and $k\left(V^{\infty}\right)$ is finite.

From Lemmas 3.4, 3.5 and 3.7, we have the following theorem which describes small essential spectral radius perturbations of semi-regular operators.

Theorem 3.8. Suppose that $T \in \mathcal{B}(X)$ is semi-regular. If $S \in \mathcal{B}(X), S T=T S$ and $V=$ $S+T$, then:

(a) if $r_{e}(S)<r_{-}(\widehat{T})$, then

$$
\operatorname{dim}\left(R\left(T^{\infty}\right)+R\left(V^{\infty}\right)\right) / R\left(V^{\infty}\right)<\infty ;
$$

(b) if $r_{e}(S)<r_{+}(\widetilde{T})$, then

$$
\operatorname{dim}\left(\overline{N\left(T^{\infty}\right)}+\overline{N\left(V^{\infty}\right)}\right) / \overline{N\left(T^{\infty}\right)}<\infty .
$$

If $r_{e}(S)<\min \left(r_{-}(\widehat{T}), r_{+}(\widetilde{T})\right)$, then:

(c) $V$ is essentially semi-regular.

Further, if $r_{e}(S)<\min \left(s_{\mathrm{sur}}(\widehat{T}), s_{\mathrm{inj}}(\widetilde{T})\right)$, then:

(d) $\operatorname{dim} R\left(V^{n}\right) / R\left(V^{n+1}\right)=\operatorname{dim} R\left(T^{m}\right) / R\left(T^{m+1}\right)$ for all $m \geq 0$ and sufficiently large $n$;

(e) $\operatorname{dim} N\left(V^{n+1}\right) / N\left(V^{n}\right)=\operatorname{dim} N\left(T^{m+1}\right) / N\left(T^{m}\right)$ for all $m \geq 0$ and sufficiently large $n$.

Proof. (a), (b) By Lemma 3.5(c).

(c) Here we consider the map $T_{\overline{N\left(T^{\infty}\right)}}$ and $V_{\overline{N\left(T^{\infty}\right)}}$ induced by $T$ and $V$ on $X / \overline{N\left(T^{\infty}\right)}$, respectively. By Proposition 2.2, $T_{\overline{N\left(T^{\infty}\right)}}$ is bounded below, and by Proposition 2.6, $r_{e}\left(S_{\overline{N\left(T^{\infty}\right)}}\right) \leq r_{e}(S)<r_{+}\left(T_{\overline{N\left(T^{\infty}\right)}}\right)$. Thus from Lemma 3.4(a) we know that $V_{\overline{N\left(T^{\infty}\right)}}$ is an upper semi-Browder operator, and hence the subspace $R\left(V_{\overline{N\left(T^{\infty}\right)}}\right)=(R(V)+$ $\left.\overline{N\left(T^{\infty}\right)}\right) / \overline{N\left(T^{\infty}\right)}$ is closed. By (3.1), $\overline{N\left(T^{\infty}\right)} \subseteq R(V)$, so $R(V)$ is closed.

By (3.2), $N\left(V^{\infty}\right) \subseteq R\left(T^{\infty}\right)$, then from (a) we know that $k\left(V^{\infty}\right)=\operatorname{dim}\left(R\left(V^{\infty}\right)+\right.$ $\left.N\left(V^{\infty}\right)\right) / R\left(V^{\infty}\right) \leq \operatorname{dim}\left(R\left(T^{\infty}\right)+R\left(V^{\infty}\right)\right) / R\left(V^{\infty}\right)<\infty$. Consequently, $V$ is essentially semi-regular. 
(d) For $M$ in (3.1), we consider the operators $T_{M}$ and $V_{M}$ induced by $T$ and $V$ on $X / M$. From (3.1) and the semi-regularity of $T$, we can get that $T_{M}$ is bounded below. It follows from the assumption and Proposition 2.6 that $r_{e}\left(S_{M}\right) \leq r_{e}(S)<s_{\text {inj }}(\widetilde{T})$. And by Lemma 3.7(a) we know that $s_{\text {inj }}(\widetilde{T}) \leq s_{\text {inj }}\left(T_{M}\right) \leq r_{+}\left(T_{M}\right)$, then we have that $r_{e}\left(S_{M}\right)<r_{+}\left(T_{M}\right)$.

Recall that $\operatorname{dim} R\left(T^{m}\right) / R\left(T^{m+1}\right)$ is constant for $m \geq 0$, since $T$ is semi-regular. To complete the proof of (d), we consider the following two cases.

Case I. Suppose that $\operatorname{dim}(X / R(T))<\infty$. Since $s_{\text {sur }}(\widehat{T}) \leq r_{-}(\widehat{T})$, then by Lemma 3.5(a) we have that for some integer $p$, the closed subspace $M=V^{p}\left(R\left(T^{\infty}\right)\right)$ satisfies $M=V(M)=T^{-1}(M)=T(M)$. Since $s_{\text {inj }}(\widetilde{T}) \leq r_{+}(\widetilde{T})$, then by Lemma 3.5(b) we have $N\left(V^{n}\right) \subseteq R\left(T^{\infty}\right)$. Thus, for all $n \geq p, V^{-n}(M)=V^{-n}\left(V^{p}\left(R\left(T^{\infty}\right)\right)\right)=V^{-n}\left(V^{n}\left(R\left(T^{\infty}\right)\right)\right)=$ $N\left(V^{n}\right)+R\left(T^{\infty}\right)=R\left(T^{\infty}\right)$. Hence from Lemma 3.4(a) it follows that $\operatorname{dim}\left(R\left(T^{\infty}\right) / M\right)-$ $\operatorname{dim}\left(X / R\left(V^{n}\right)\right)=\alpha\left(V_{M}^{n}\right)-\beta\left(V_{M}^{n}\right)=\operatorname{ind}\left(V_{M}^{n}\right)=\operatorname{ind}\left(T_{M}^{n}\right)=(-n) \cdot \operatorname{dim}(X / R(T))$ for all $n \geq p$. Then, we can conclude that $\operatorname{dim} R\left(V^{n}\right) / R\left(V^{n+1}\right)=\operatorname{ind}\left(V_{M}^{n}\right)-\operatorname{ind}\left(V_{M}^{n+1}\right)=$ $\operatorname{ind}\left(T_{M}^{n}\right)-\operatorname{ind}\left(T_{M}^{n+1}\right)=\operatorname{dim}(X / R(T))$ for all $n \geq p$.

Case II. Suppose that $\operatorname{dim}(X / R(T))=\infty$. By Lemma 3.4(a),

$$
\operatorname{ind}\left(V_{M}\right)=\operatorname{ind}\left(T_{M}\right)=-\beta\left(T_{M}\right)=-\operatorname{dim}(X / R(T))=-\infty,
$$

then $\beta\left(V_{M}\right)=\infty$, that is, $X / R(V)$ is infinite-dimensional. For all $n \in \mathbb{N}$, the map induced by $V^{n}$ from $X / R(V)$ to $R\left(V^{n}\right) / R\left(V^{n+1}\right)$ is onto, and its null space is $\left(N\left(V^{n}\right)+\right.$ $R(V)) / R(V)$. From Lemma 3.5(c) we can infer that $\operatorname{dim}\left(\left(N\left(V^{n}\right)+R(V)\right) / R(V)\right)<\infty$, and thus $R\left(V^{n}\right) / R\left(V^{n+1}\right)$ is infinite-dimensional for all $n \in \mathbb{N}$.

(e) For $L$ in (3.2), we consider the restrictions $\left.T\right|_{L}$ and $\left.V\right|_{L}$. From (3.2), we know that $\left.T\right|_{L}$ is onto. It follows from the assumption and Proposition 2.6 that $r_{e}\left(\left.S\right|_{L}\right) \leq$ $r_{e}(S)<s_{\text {sur }}(\widehat{T})$. And by Lemma 3.7(b) we know that $s_{\text {sur }}(\widehat{T}) \leq s_{\text {sur }}\left(\left.T\right|_{L}\right) \leq r_{-}\left(\left.T\right|_{L}\right)$, then we have that $r_{e}\left(\left.S\right|_{L}\right)<r_{-}\left(\left.T\right|_{L}\right)$.

Recall that $\operatorname{dim} N\left(T^{m+1}\right) / N\left(T^{m}\right)$ is constant for $m \geq 0$, since $T$ is semi-regular. To complete the proof of (e), we consider the following two cases.

Case I. Suppose that $\operatorname{dim} N\left(T^{m+1}\right) / N\left(T^{m}\right)=\operatorname{dim} N(T) \cap R\left(T^{m}\right)=\operatorname{dim} N(T)<\infty$. Since $s_{\text {inj }}(\widetilde{T}) \leq r_{+}(\widetilde{T})$, then from Lemma 3.5(b) it follows that for some integer $q$, the closed subspace $L=V^{-q}\left(\overline{N\left(T^{\infty}\right)}\right)$ satisfies $L=V^{-1}(L)=T(L)=T^{-1}(L)$. Since $s_{\text {sur }}(\widehat{T}) \leq r_{-}(\widehat{T})$, from Lemma 3.5(a), we have $\overline{N\left(T^{\infty}\right)} \subseteq R\left(V^{n}\right)$. Thus, for all $n \geq q$,

$$
V^{n}(L)=V^{n}\left(V^{-q}\left(\overline{N\left(T^{\infty}\right)}\right)\right)=V^{n}\left(V^{-n}\left(\overline{N\left(T^{\infty}\right)}\right)\right)=R\left(V^{n}\right) \cap \overline{N\left(T^{\infty}\right)}=\overline{N\left(T^{\infty}\right)} .
$$

Hence, by Lemma 3.4(b),

$$
\begin{aligned}
\operatorname{dim} N\left(V^{n}\right)-\operatorname{dim}\left(L / \overline{N\left(T^{\infty}\right)}\right) & =\operatorname{dim}\left(N\left(V^{n}\right) \cap L\right)-\operatorname{dim}\left(L / \overline{N\left(T^{\infty}\right)}\right) \\
& =\alpha\left(\left.V^{n}\right|_{L}\right)-\beta\left(\left.V^{n}\right|_{L}\right) \\
& =\operatorname{ind}\left(\left.V^{n}\right|_{L}\right)=\operatorname{ind}\left(\left.T^{n}\right|_{L}\right)=\operatorname{dim}\left(N\left(T^{n}\right) \cap L\right) \\
& =\operatorname{dim} N\left(T^{n}\right)
\end{aligned}
$$


for all $n \geq q$. And then we can conclude that $\operatorname{dim} N\left(V^{n+1}\right) / N\left(V^{n}\right)=\operatorname{ind}\left(\left.V^{n+1}\right|_{L}\right)-$ $\operatorname{ind}\left(\left.V^{n}\right|_{L}\right)=\operatorname{ind}\left(\left.T^{n+1}\right|_{L}\right)-\operatorname{ind}\left(\left.T^{n}\right|_{L}\right)=\operatorname{dim}(N(T))$ for all $n \geq q$.

Case II. Suppose that $\operatorname{dim} N\left(T^{m+1}\right) / N\left(T^{m}\right)=\infty$. By Lemma 3.4(b), ind $\left(\left.V\right|_{L}\right)=$ $\operatorname{ind}\left(\left.T\right|_{L}\right)=\operatorname{dim}(N(T) \cap L)=\operatorname{dim} N(T)=\infty$, then $\alpha\left(\left.V\right|_{L}\right)=\infty$, that is, $N(V)=$ $N(V) \cap L$ is infinite-dimensional. For all $n \in \mathbb{N}$, the map induced by $V^{n}$ from $N\left(V^{n+1}\right) / N\left(V^{n}\right)$ to $N(V)$ is one-to-one, and its cokernel is $N(V) /\left(N(V) \cap R\left(V^{n}\right)\right)$. From Lemma 3.5(c) we can infer that $N(V) /\left(N(V) \cap R\left(V^{n}\right)\right)$ is finite-dimensional, and thus $N\left(V^{n+1}\right) / N\left(V^{n}\right)$ is infinite-dimensional for all $n \in \mathbb{N}$.

The next theorem describes arbitrary small essential spectral radius perturbations of an operator with topological uniform descent.

Theorem 3.9. Suppose that $T \in \mathcal{B}(X)$ has topological uniform descent for $m \geq d \neq 0$. If $S \in \mathcal{B}(X), S T=T S$ and $V=S+T$, then:

(a) if $r_{e}(S)<r_{-}(\widehat{T})$, then

$$
\operatorname{dim}\left(R\left(T^{\infty}\right)+R\left(V^{\infty}\right)\right) / R\left(V^{\infty}\right)<\infty
$$

(b) if $r_{e}(S)<r_{+}(\widetilde{T})$, then

$$
\operatorname{dim}\left(\overline{N\left(T^{\infty}\right)}+\overline{N\left(V^{\infty}\right)}\right) / \overline{N\left(T^{\infty}\right)}<\infty ;
$$

if $r_{e}\left(\left.S\right|_{R\left(T^{d}\right)}\right)<\min \left(s_{\mathrm{sur}}\left(\widehat{T_{d}}\right), s_{\mathrm{inj}}\left(\widetilde{T_{d}}\right)\right)$, then:

(c) $\operatorname{dim} R\left(V^{n}\right) / R\left(V^{n+1}\right) \geq \operatorname{dim} R\left(T^{m}\right) / R\left(T^{m+1}\right)$ for all $m \geq d$ and sufficiently large $n$;

(d) $\operatorname{dim} N\left(V^{n+1}\right) / N\left(V^{n}\right) \geq \operatorname{dim} N\left(T^{m+1}\right) / N\left(T^{m}\right)$ for all $m \geq d$ and sufficiently large $n$.

Proof. (a), (b) By Lemma 3.6(c).

(c), (d) Recall that $\operatorname{dim} R\left(T^{m}\right) / R\left(T^{m+1}\right)$ and $\operatorname{dim} N\left(T^{m+1}\right) / N\left(T^{m}\right)$ are constant for $m \geq d$, since $T$ has uniform descent for $m \geq d$. Hence part (c) follows directly from Theorem 3.8(d) and Proposition 2.4(a); and part (d) follows directly from Theorem 3.8(e) and Proposition 2.4(b).

In order to replace the inequalities in parts (c) and (d) of Theorem 3.9 with equalities, we need additional hypotheses on $S$ or $V$. For a spectral analysis of $T$, the most important case is when $S=\lambda I$. Since the proof is no harder under the assumption that $S$ is invertible, rather than a scalar multiple of the identity, we use this assumption as the added hypothesis on $S$.

Theorem 3.10. Suppose that $T \in \mathcal{B}(X)$ has topological uniform descent for $m \geq d \neq 0$. If $S \in \mathcal{B}(X)$ is invertible, $S T=T S, r_{e}\left(\left.S\right|_{R\left(T^{d}\right)}\right)<\min \left(s_{\text {sur }}\left(\widehat{T_{d}}\right), s_{\mathrm{inj}}\left(\widetilde{T_{d}}\right)\right)$ and $V=S+T$, then:

(a) $\operatorname{dim} R\left(V^{n}\right) / R\left(V^{n+1}\right)=\operatorname{dim} R\left(T^{m}\right) / R\left(T^{m+1}\right)$ for all $m \geq d$ and sufficiently large $n$;

(b) $\operatorname{dim} N\left(V^{n+1}\right) / N\left(V^{n}\right)=\operatorname{dim} N\left(T^{m+1}\right) / N\left(T^{m}\right)$ for all $m \geq d$ and sufficiently large $n$.

Proof. Define by induction the following sequence: $R_{1}=-S^{-1}, R_{n+1}=S^{-1} V R_{n}+$ $R_{1}(n \geq 1)$. From the invertibility of $S$ and $T S=S T$, we can infer that $T R_{n}=R_{n} T$, $S R_{n}=R_{n} S$ and $S^{-n} V^{n}=I-T R_{n}$ for all $n \in \mathbb{N}$. 
Indeed, when $n=1$, then $R_{1}=-S^{-1}$ satisfies $T R_{1}=R_{1} T, S R_{1}=R_{1} S$ and $S^{-1} V=$ $S^{-1}(S+T)=I+S^{-1} T=I-R_{1} T=I-T R_{1}$. Suppose that $T R_{k}=R_{k} T, S R_{k}=R_{k} S$ and $S^{-k} V^{k}=I-T R_{k}$ hold. Then, when $n=k+1$,

$$
\begin{gathered}
T R_{k+1}=T\left(S^{-1} V R_{k}+R_{1}\right)=\left(S^{-1} V R_{k}+R_{1}\right) T=R_{k+1} T, \\
S R_{k+1}=S\left(S^{-1} V R_{k}+R_{1}\right)=\left(S^{-1} V R_{k}+R_{1}\right) S=R_{k+1} S
\end{gathered}
$$

and

$$
\begin{aligned}
S^{-(k+1)} V^{k+1}=S^{-1}\left(S^{-k} V^{k}\right) V & =S^{-1}\left(I-T R_{k}\right) V=S^{-1} V-S^{-1} T R_{k} V \\
& \left.=\left(I-T R_{1}\right)-T S^{-1} V R_{k}\right)=I-T\left(S^{-1} V R_{k}+R_{1}\right) \\
& =I-T R_{k+1} .
\end{aligned}
$$

Hence it follows that $N\left(V^{\infty}\right) \subseteq R\left(T^{\infty}\right)$, and by interchanging the roles of $V$ and $T$ we also have $N\left(T^{\infty}\right) \subseteq R\left(V^{\infty}\right)$. Parts (a) and (b) now follow immediately from Theorem 3.8(d) and (e) and Proposition 2.4(a) and (b).

Corollary 3.11. Suppose that $T \in \mathcal{B}(X)$ has topological uniform descent for $m \geq$ $d \neq 0 . \quad$ If $S \in \mathcal{B}(X)$ is invertible, $S T=T S, \quad r_{e}\left(\left.S\right|_{R\left(T^{d}\right)}\right)<\min \left(s_{\mathrm{sur}}\left(\widehat{T_{d}}\right), s_{\mathrm{inj}}\left(\widetilde{T_{d}}\right)\right)$ and $V=S+T$, then:

(a) $\operatorname{asc}(V)<\infty \Longleftrightarrow \operatorname{asc}(T)<\infty, \quad \operatorname{asc}_{e}(V)<\infty \Longleftrightarrow \operatorname{asc}_{e}(T)<\infty$;

(b) $\operatorname{dsc}(V)<\infty \Longleftrightarrow \operatorname{dsc}(T)<\infty, \quad \operatorname{dsc}_{e}(V)<\infty \Longleftrightarrow \operatorname{dsc}_{e}(T)<\infty$.

We can improve Theorem 3.9(a), (b) and replace the inequalities in Theorem 3.9(c), (d) with equalities if we assume that both $T$ and $V$ have topological uniform descent. We do this in the following theorem.

Theorem 3.12. Suppose that $T \in \mathcal{B}(X)$ has topological uniform descent for $m \geq d \neq 0$. If $S \in \mathcal{B}(X)$, $S T=T S$ and $V=S+T$ has topological uniform descent for $n \geq l$, then:

(a) if $r_{e}(S)<\min \left(r_{-}(\widehat{T}), r_{-}(\widehat{V})\right)$, then

$$
\operatorname{dim}\left(R\left(T^{\infty}\right)+R\left(V^{\infty}\right)\right) /\left(R\left(T^{\infty}\right) \cap R\left(V^{\infty}\right)\right)<\infty ;
$$

(b) if $r_{e}(S)<\min \left(r_{+}(\widetilde{T}), r_{+}(\widetilde{V})\right)$ then

$$
\begin{aligned}
& \qquad \operatorname{dim}\left(\overline{N\left(T^{\infty}\right)}+\overline{N\left(V^{\infty}\right)}\right) /\left(\overline{N\left(T^{\infty}\right)} \cap \overline{N\left(V^{\infty}\right)}\right)<\infty ; \\
& \quad \text { if } r_{e}\left(\left.S\right|_{R\left(T^{d}\right)}\right)<\min \left(s_{\mathrm{sur}}\left(\widehat{T_{d}}\right), s_{\mathrm{inj}}\left(\widetilde{T_{d}}\right)\right) \text { and } r_{e}\left(\left.S\right|_{R\left(V^{l}\right)}\right)<\min \left(s_{\mathrm{sur}}\left(\widehat{V}_{l}\right), s_{\mathrm{inj}}\left(\widetilde{V}_{l}\right)\right) \text {, } \\
& \text { then: } \\
& \text { (c) } \operatorname{dim} R\left(V^{n}\right) / R\left(V^{n+1}\right)=\operatorname{dim} R\left(T^{m}\right) / R\left(T^{m+1}\right) \text { for sufficiently large } m \text { and } n ; \\
& \text { (d) } \operatorname{dim} N\left(V^{n+1}\right) / N\left(V^{n}\right)=\operatorname{dim} N\left(T^{m+1}\right) / N\left(T^{m}\right) \text { for sufficiently large } m \text { and } n .
\end{aligned}
$$

Proof. Note that if $M$ and $N$ are linear subspaces of $X$, then by [12, Lemma 2.2] we have $(M+N) / N \approx M /(M \cap N)$. Hence parts (a) and (b) follow directly from Theorem 3.9(a) and (b).

Parts (c) and (d) are immediate consequences of Theorem 3.9(c) and (d).

In order to have Theorem 3.12 apply to all commuting small essential spectral radius perturbations of an operator $T$ with topological uniform descent, we need to consider 
those $T$ for which all small essential spectral radius perturbations have topological uniform descent. That some restriction on $T$ is necessary is clear from considering the special cases in which $T$ is the zero operator or is a projection of finite rank. We see in the next theorem that when $T$ is essentially semi-regular, then $V$ is also essentially semi-regular.

Let $T \in \mathcal{B}(X)$. Note that if $k_{d}(T)=0$ for some $d \in \mathbb{N}$, then $k_{n}(T)=0$ for all $n \geq d$. In addition, if $T$ is essentially semi-regular, then $T^{n}$ is essentially semi-regular, and hence $R\left(T^{n}\right)$ is closed for all $n \in \mathbb{N}$ (see [1, Theorem 1.51]). Hence it is easy to verify that if $T \in \mathcal{B}(X)$ is essentially semi-regular $(d:=k(T))$, then $T$ has topological uniform descent for $n \geq d$.

Theorem 3.13. Suppose that $T \in \mathcal{B}(X)$ is essentially semi-regular $(d:=k(T))$. If $S \in$ $\mathcal{B}(X), S T=T S, r_{e}(S)<\min \left(r_{-}(\widehat{T}), r_{+}(\widetilde{T})\right)$ and $r_{e}\left(\left.S\right|_{R\left(T^{d}\right)}\right)<\min \left(r_{-}\left(\widehat{T_{d}}\right), r_{+}\left(\widetilde{T_{d}}\right)\right)$, then $V=S+T$ is essentially semi-regular.

Proof. We first prove that $k\left(V^{\infty}\right)<\infty$. Since $T$ is essentially semi-regular, then $T$ has uniform descent for $n \geq d$, where $d=k(T)$. Since $r_{e}(S)<r_{+}(\widetilde{T})$, by Lemma 3.6(b), there exists a closed subspace $L$ satisfying $L=V^{-1}(L)=T(L)+\overline{N\left(T^{\infty}\right)}$, and then we have

$$
\begin{aligned}
N\left(V^{\infty}\right) \subseteq L & =T(L)+\overline{N\left(T^{\infty}\right)}=T\left(T(L)+\overline{N\left(T^{\infty}\right)}\right)+\overline{N\left(T^{\infty}\right)} \\
& =T^{2}(L)+T\left(\overline{N\left(T^{\infty}\right)}\right)+\overline{N\left(T^{\infty}\right)}=T^{2}(L)+\overline{N\left(T^{\infty}\right)} \\
& =T^{2}\left(T(L)+\overline{N\left(T^{\infty}\right)}\right)+\overline{N\left(T^{\infty}\right)}=\cdots \\
& =R\left(\left(\left.T\right|_{L}\right)^{\infty}\right)+\overline{N\left(T^{\infty}\right)} \subseteq R\left(T^{\infty}\right)+\overline{N\left(T^{\infty}\right)} .
\end{aligned}
$$

By Proposition 2.3, $R\left(T^{\infty}\right)+\overline{N\left(T^{\infty}\right)}=R\left(T^{\infty}\right)+N\left(T^{\infty}\right)$. Since $T$ is essentially semiregular, $k\left(T^{\infty}\right)=\operatorname{dim}\left(R\left(T^{\infty}\right)+N\left(T^{\infty}\right)\right) / R\left(T^{\infty}\right)<\infty$, and hence there exists a finitedimensional subspace $F$ of $X$ such that $N\left(T^{\infty}\right) \subseteq R\left(T^{\infty}\right)+F$. Since $r_{e}(S)<r_{-}(\widehat{T})$, by Lemma 3.6(c), then $\operatorname{dim}\left(R\left(T^{\infty}\right)+R\left(V^{\infty}\right)\right) / R\left(V^{\infty}\right)<\infty$, and hence

$$
\begin{aligned}
k\left(V^{\infty}\right) & =\operatorname{dim}\left(N\left(V^{\infty}\right)+R\left(V^{\infty}\right)\right) / R\left(V^{\infty}\right) \\
& \leq \operatorname{dim}\left(R\left(T^{\infty}\right)+\overline{N\left(T^{\infty}\right)}+R\left(V^{\infty}\right)\right) / R\left(V^{\infty}\right) \\
& =\operatorname{dim}\left(R\left(T^{\infty}\right)+N\left(T^{\infty}\right)+R\left(V^{\infty}\right)\right) / R\left(V^{\infty}\right) \\
& \leq \operatorname{dim}\left(R\left(T^{\infty}\right)+R\left(V^{\infty}\right)+F\right) / R\left(V^{\infty}\right)<\infty .
\end{aligned}
$$

Next we show that $R(V)$ is closed. Since $T_{d}=\left.T\right|_{R\left(T^{d}\right)}$ is semi-regular and $r_{e}\left(\left.S\right|_{R\left(T^{d}\right)}\right)<\min \left(r_{-}\left(\widehat{T_{d}}\right), r_{+}\left(\widetilde{T_{d}}\right)\right)$, then from Theorem 3.8(c) we know that $R\left(V T^{d}\right)$ is closed in the operator range topology on $R\left(T^{d}\right)$. Since $T^{d}$ induces a bounded operator from $X$ to $R\left(T^{d}\right)$, with the operator range topology, then by Proposition 2.1(b) we have that $T^{-d}\left(R\left(T^{d} V\right)\right)$ is closed in $X$. From Proposition 2.4 we know that the map induced by $T^{d}$ from $X / R(V)$ to $R\left(T^{d}\right) / R\left(T^{d} V\right)$ is onto, and its kernel is isomorphic to $N\left(T^{d}\right) /\left(R(V) \cap N\left(T^{d}\right)\right)$. Since $R(V) \supseteq R\left(V^{\infty}\right)$,

$$
\begin{aligned}
\operatorname{dim} N\left(T^{d}\right) /\left(R(V) \cap N\left(T^{d}\right)\right) & \leq \operatorname{dim} N\left(T^{d}\right) /\left(R\left(V^{\infty}\right) \cap N\left(T^{d}\right)\right) \\
& =\operatorname{dim}\left(N\left(T^{d}\right)+R\left(V^{\infty}\right)\right) / R\left(V^{\infty}\right)
\end{aligned}
$$




$$
\begin{aligned}
& \leq \operatorname{dim}\left(N\left(T^{\infty}\right)+R\left(V^{\infty}\right)\right) / R\left(V^{\infty}\right) \\
& \leq \operatorname{dim}\left(R\left(T^{\infty}\right)+R\left(V^{\infty}\right)+F\right) / R\left(V^{\infty}\right)<\infty .
\end{aligned}
$$

Hence $R(V)$ has finite codimension in $T^{-d}\left(R\left(T^{d} V\right)\right)$. By [1, Corollary 1.15], $R(V)$ is closed.

Remark 3.14. Suppose that $T \in \mathcal{B}(X)$ is essentially semi-regular $(d:=k(T))$, and that $S \in \mathcal{B}(X)$ commutes with $T$. Note that $\widehat{T}=\widehat{T_{d}}$ and that $r_{e}\left(\left.S\right|_{R\left(T^{d}\right)}\right) \leq r_{e}(S)$ (see Theorem 4.4 below). Then the condition that $r_{e}(S)<\min \left(r_{-}(\widehat{T}), r_{+}(\widetilde{T})\right.$ ) and $r_{e}\left(\left.S\right|_{R\left(T^{d}\right)}\right)<\min \left(r_{-}\left(\widehat{T_{d}}\right), r_{+}\left(\widetilde{T_{d}}\right)\right)$ in Theorem 3.13 can be replaced by $r_{e}(S)<$ $\min \left(r_{-}(\widehat{T}), r_{+}(\widetilde{T}), r_{+}\left(\widetilde{T_{d}}\right)\right)$.

A result similar to Theorem 3.13 was proved by Kordula et al. in [14, Theorem 16], in which the condition is $r_{e}(S)<\min \left(r_{-}(\widehat{T}), r_{+}\left(T_{R\left(T^{\infty}\right)}\right)\right)$.

By Theorems 3.12 and 3.13, we immediately have the following result.

Theorem 3.15. Suppose that $T \in \mathcal{B}(X)$ is essentially semi-regular $(d:=k(T))$. If $S \in$ $\mathcal{B}(X), S T=T S, r_{e}(S)<\min \left(r_{-}(\widehat{T}), r_{+}(\widetilde{T})\right), r_{e}\left(\left.S\right|_{R\left(T^{d}\right)}\right)<\min \left(r_{-}\left(\widehat{T_{d}}\right), r_{+}\left(\widetilde{T_{d}}\right)\right)$ and $V=$ $T+S$, then:

(a) $V$ is essentially semi-regular;

(b) if $r_{e}(S)<r_{-}(\widehat{V})$, then

$$
\operatorname{dim}\left(R\left(T^{\infty}\right)+R\left(V^{\infty}\right)\right) /\left(R\left(T^{\infty}\right) \cap R\left(V^{\infty}\right)\right)<\infty ;
$$

(c) if $r_{e}(S)<r_{+}(\widetilde{V})$, then

$$
\begin{aligned}
& \qquad \operatorname{dim}\left(\overline{N\left(T^{\infty}\right)}+\overline{N\left(V^{\infty}\right)}\right) /\left(\overline{N\left(T^{\infty}\right)} \cap \overline{N\left(V^{\infty}\right)}\right)<\infty ; \\
& \text { if } r_{e}\left(\left.S\right|_{R\left(T^{d}\right)}\right)<\min \left(s_{\mathrm{sur}}\left(\widehat{T_{d}}\right), s_{\mathrm{inj}}\left(\widetilde{T_{d}}\right)\right) \text { and } r_{e}\left(\left.S\right|_{R\left(V^{l}\right)}\right)<\min \left(s_{\mathrm{sur}}\left(\widehat{V_{l}}\right), s_{\mathrm{inj}}\left(\widetilde{V}_{l}\right)\right), \\
& \text { where } l:=k(V), \text { then: } \\
& \text { (d) } \operatorname{dim} R\left(V^{n}\right) / R\left(V^{n+1}\right)=\operatorname{dim} R\left(T^{m}\right) / R\left(T^{m+1}\right) \text { for sufficiently large } m \text { and } n ; \\
& \text { (e) } \operatorname{dim} N\left(V^{n+1}\right) / N\left(V^{n}\right)=\operatorname{dim} N\left(T^{m+1}\right) / N\left(T^{m}\right) \text { for sufficiently large } m \text { and } n .
\end{aligned}
$$

\section{Some comments}

In this section we show, as we stated at the beginning of this paper, that small essential spectral radius perturbations cover compact, quasinilpotent and Riesz perturbations.

Theorem 4.1. Suppose that $T \in \mathcal{B}(X)$ is semi-regular. Then we have $\gamma(T) \leq$ $\min \left(s_{\text {sur }}(\widehat{T}), s_{\text {inj }}(\widetilde{T})\right)$.

Proof. Since $T$ is semi-regular, by mimicking the proof of Lemma 3.7, $\gamma(T) \leq$ $\min (\gamma(\widehat{T}), \gamma(\widetilde{T}))$. Since $\widehat{T}$ is onto, by $(2.4), s_{\text {sur }}(\widehat{T})=\lim _{n \rightarrow \infty} \gamma\left(\widehat{T}^{n}\right)^{1 / n} \geq \gamma(\widehat{T})$. Since $\widetilde{T}$ is bounded below, by $(2.3), s_{\text {inj }}(\widetilde{T})=\lim _{n \rightarrow \infty} \gamma\left(\widetilde{T}^{n}\right)^{1 / n} \geq \gamma(\widetilde{T})$. Hence $\gamma(T) \leq$ $\min \left(s_{\text {sur }}(\widehat{T}), s_{\text {inj }}(\widetilde{T})\right)$. 
Theorem 4.2. Suppose that $T \in \mathcal{B}(X)$ has topological uniform descent for $n \geq d$. Then $\gamma\left(T_{d}\right) \leq \min \left(s_{\text {sur }}(\widehat{T}), s_{\text {inj }}(\widetilde{T})\right)$.

Proof. Since $T$ has topological uniform descent for $n \geq d$, by [9, Theorem 3.1], we have $R\left(T^{d}\right) \cap N(T)=R\left(T^{\infty}\right) \cap N(T)$ and $N\left(T^{d}\right)+R(T)=N\left(T^{\infty}\right) \cap R(T)$. Note that $\left[T^{d}\right]^{-1}\left(\left.T\right|_{R\left(T^{d}\right)}\right)\left[T^{d}\right]=T_{N\left(T^{d}\right)}$, where $\left[T^{d}\right]: X / N\left(T^{d}\right) \rightarrow\left(R\left(T^{d}\right),\|\cdot\|_{R\left(T^{d}\right)}\right)$ is isometric and invertible. Recall that if $M$ and $N$ are linear subspaces of $X$, then $(M+N)^{\perp}=$ $M^{\perp} \cap N^{\perp}$. Now, by mimicking the proof of Lemma 3.7, we have that $\gamma\left(T_{d}\right) \leq \gamma(\widehat{T})$ and $\gamma\left(T_{d}\right) \leq \gamma(\widetilde{T})$. Since $\widehat{T}$ is onto, by (2.4), we have $s_{\text {sur }}(\widehat{T})=\lim _{n \rightarrow \infty} \gamma\left(\widehat{T}^{n}\right)^{1 / n} \geq \gamma(\widehat{T})$. Since $\widetilde{T}$ is bounded below, by (2.3), we have $s_{\text {inj }}(\widetilde{T})=\lim _{n \rightarrow \infty} \gamma\left(\widetilde{T}^{n}\right)^{1 / n} \geq \gamma(\widetilde{T})$. Hence $\gamma\left(T_{d}\right) \leq \min \left(s_{\text {sur }}(\widehat{T}), s_{\text {inj }}(\widetilde{T})\right)$.

THEOREM 4.3. Suppose that $T$ and $S$ are commuting bounded operators on the Banach space $X$, and that $S$ is lower semi-Fredholm, then $\left.S\right|_{R(T)}:\left(R(T),\|\cdot\|_{R(T)}\right) \rightarrow$ $\left(R(T),\|\cdot\|_{R(T)}\right)$ is also lower semi-Fredholm.

Proof. Since $S$ is lower semi-Fredholm, $S^{*}$ is upper semi-Fredholm, and hence $\left.S^{*}\right|_{N(T)^{\perp}}$ is also upper semi-Fredholm. It follows that $\left(S_{N(T)}\right)^{*}=\left.Q^{-1} S^{*}\right|_{N(T)^{\perp}} Q$ is upper semi-Fredholm, where $Q:(X / N(T))^{*} \rightarrow N(T)^{\perp}$ is isometric and invertible. Let $[T]: X / N(T) \rightarrow\left(R(T),\|\cdot\|_{R(T)}\right)$ be an isometric and invertible operator. Since $[T]^{-1}\left(\left.S\right|_{R(T)}\right)[T]=S_{N(T)}$, then $[T]^{*}\left(\left.S\right|_{R(T)}\right)^{*}\left([T]^{*}\right)^{-1}=\left(S_{N(T)}\right)^{*}$ is upper semiFredholm, and hence $[T]^{-1}\left(\left.S\right|_{R(T)}\right)[T]=S_{N(T)}$ is lower semi-Fredholm. Thus $\left.S\right|_{R(T)}$ is also lower semi-Fredholm.

Let $T \in \mathcal{B}(X)$. Denote the upper semi-Fredholm spectrum, lower semiFredholm spectrum and the essential spectrum of $T$ by $\sigma_{+}(T)=\{\lambda \in \mathbb{C}: T-\lambda I$ is not upper semi-Fredholm $\}, \sigma_{-}(T)=\{\lambda \in \mathbb{C}: T-\lambda I$ is not lower semi-Fredholm $\}$ and $\sigma_{e}(T)=\{\lambda \in \mathbb{C}: T-\lambda I$ is not Fredholm $\}$, respectively. Note that $r_{e}(T)=\max \{|\lambda|$ : $\left.\lambda \in \sigma_{e}(T)\right\}$ and that the upper (respectively, lower) semi-Fredholm spectrum of $T$ contains the boundary of the essential spectrum of $T$ (see [11, Theorem 3]).

Next, we present a useful result concerning operator ranges. The reader should compare it with Proposition 2.6.

THEOREM 4.4. Suppose that $T$ and $S$ are commuting bounded operators on the Banach space $X$. Then $r_{e}\left(\left.S\right|_{R(T)}\right) \leq r_{e}(S)$.

Proof. For any $T \in \mathcal{B}(X)$, note that $r_{e}(T)=\max \left\{|\lambda|: \lambda \in \sigma_{e}(T)\right\}$ and that the lower semi-Fredholm spectrum of $T$ contains the boundary of the essential spectrum of $T$. Then the desired conclusion follows from Theorem 4.3.

Remark 4.5. Suppose that $T \in \mathcal{B}(X)$ has topological uniform descent for $n \geq d$, and that $S \in \mathcal{B}(X)$ commutes with $T$. Then by Proposition 3.2 and Theorems $4.1,4.2$ and 4.4, we have the following inequalities:

$$
\begin{gathered}
\left\|\left.S\right|_{R\left(T^{d}\right)}\right\|_{R\left(T^{d}\right)} \leq\|S\| ; \\
\gamma\left(T_{d}\right) \leq \min \left(s_{\mathrm{sur}}\left(\widehat{T_{d}}\right), s_{\mathrm{inj}}\left(\widetilde{T_{d}}\right)\right) ; \\
\gamma\left(T_{d}\right) \leq \min \left(s_{\mathrm{sur}}(\widehat{T}), s_{\mathrm{inj}}(\widetilde{T})\right) ; \\
r_{e}\left(\left.S\right|_{R\left(T^{d}\right)}\right) \leq r_{e}(S) .
\end{gathered}
$$


Inequality (4.1) seems to have been discovered by Grabiner in the fourth paragraph of [9, Section 4], although he states that $\|S\| \leq\left\|\left.S\right|_{R\left(T^{d}\right)}\right\|_{R\left(T^{d}\right)}$ (which, in general, is not true). Recall that in [9], by sufficiently small Grabiner means that the norm of the restriction of $S$ to $R\left(T^{d}\right)$ is less than the reduced minimum modulus of the restriction of $T$ to $R\left(T^{d}\right)$. Hence by (4.2) and (4.3), one can readily see that our perturbational results extend some sufficiently small perturbational results of Grabiner [9].

Note that $S$ is Riesz if and only if $r_{e}(S)=0$. Hence by (4.4), one can readily see that small essential spectrum radius perturbations cover Riesz perturbations. In particular, small essential spectrum radius perturbations cover compact perturbations in [9] and quasinilpotent perturbations. Consequently, our perturbational results extend all compact perturbational results of Grabiner [9].

As far as we know, most of our perturbations results are new even for Fredholm operators.

Finally, from the proof of Theorem 4.3, we get the following results concerning operator ranges.

Corollary 4.6. Suppose that $T$ and $S$ are commuting bounded operators on the Banach space $X$. If $S$ is quasi-nilpotent, compact, or Riesz, respectively, then $\left.S\right|_{R(T)}$ has the same property. If $S$ has any one of the above properties and if $T$ has infinite rank, then $R(S T)$ has infinite codimension in $R(T)$.

Proof. The first statement follows from the proof of Theorem 4.3, since the properties described in Corollary 4.6 are conjugate invariance, similarity invariance and inherited by the restrictions on closed invariant subspaces. Note that the lower semi-Fredholm spectrum of an operator on an infinite-dimensional Banach space is nonempty. Then the second statement follows immediately from the first statement.

From the fact that the upper semi-Fredholm spectrum of an operator on an infinitedimensional Banach space is nonempty, we immediately have the following corollary, which is the dual of Corollary 4.6 for kernels.

Corollary 4.7. Suppose that $T$ and $S$ are commuting bounded operators on the Banach space $X$. If $S$ is Riesz, $T$ has infinite rank, and $R(S T)$ is closed in the operator range topology on $R(T)$, then $N(T)$ has infinite codimension in $N(S T)$.

Proof. By Corollary 4.6, $\left.S\right|_{R(T)}$ is Riesz. Note that the lower semi-Fredholm spectrum of an operator on an infinite-dimensional Banach space is nonempty, and that $N(S T) / N(T)$ is linearly isomorphic to $N(S) \cap R(T)$. The desired conclusion follows immediately.

Remark 4.8. Corollary 4.6 was proved by Grabiner in [8, Theorem 4.2], but our proof uses a rather direct technique different from [8]. A result similar to Corollary 4.7 is also proved by Grabiner in [8, Corollary 4.3], in which the condition that $R(S T)$ is closed in the operator range topology on $R(T)$ is replaced by $R(S T)$ being closed in $X$. Here we note that, by [10, Theorem 1], the condition that $R(S T)=R(T S)$ is closed in $X$ implies that $N(T)+R(S)$ is closed in $X$. And by Proposition 2.1, this last 
condition is equivalent to $R(S T)$ being closed in the operator range topology on $R(T)$. In conclusion, the condition that $R(S T)$ is closed in $X$ implies that $R(S T)$ is closed in the operator range topology on $R(T)$. But the reverse is not true. We see this by taking $S=I$ and $T$ with nonclosed range $R(T)$.

\section{References}

[1] P. Aiena, Fredholm and Local Spectral Theory, with Application to Multipliers (Kluwer Academic Publishers, Dordrecht, 2004).

[2] P. Aiena, M. T. Biondi and C. Carpintero, 'On Drazin invertibility', Proc. Amer. Math. Soc. 136(8) (2008), 2839-2848.

[3] O. Bel Hadj Fredj, M. Burgos and M. Oudghiri, 'Ascent spectrum and essential ascent spectrum', Studia Math. 187(1) (2008), 59-73.

[4] M. Berkani, 'Restrition of operator to the range of its power', Studia Math. 140(2) (2000), 163-175.

[5] M. Berkani and M. Sarih, 'On semi-B-Fredholm operators', Glasg. Math. J. 43(3) (2001), 457-465.

[6] M. Burgos, A. Kaidi, M. Mbekhta and M. Oudghiri, 'The descent spectrum and perturbations', J. Operator Theory 56(2) (2006), 259-271.

[7] P. A. Fillmore and J. P. Williams, 'On operator ranges', Adv. Math. 7(3) (1971), 254-281.

[8] S. Grabiner, 'Ranges of products of operators', Canad. J. Math. XXVI(6) (1974), 1430-1441.

[9] S. Grabiner, 'Uniform ascent and descent of bounded operators', J. Math. Soc. Japan 34(2) (1982), 317-337.

[10] R. E. Harte, 'On Kato non-singularity', Studia Math. 177(2) (1996), 107-114.

[11] R. E. Harte and A. Wickstead, 'Upper and lower Fredholm spectra II', Math. Z. 154(3) (1997), 253-256.

[12] M. A. Kaashoek, 'Ascent, descent, nullity and defect, a note on a paper by A. E. Taylor', Math. Ann. 172(2) (1967), 105-115.

[13] V. Kordula and V. Müller, 'The distance from the Apostol spectrum', Proc. Amer. Math. Soc. 124(10) (1996), 3055-3061.

[14] V. Kordula, V. Müller and V. Rakočevič, 'On the semi-Browder spectrum', Studia Math. 123(1) (1997), 1-13.

[15] E. Makai and J. Zemánek, 'The surjectivity radius, packing numbers and boundedness below of linear operators', Integral Equations Operator Theory 6(1) (1983), 372-384.

[16] M. Mbekhta and V. Müller, 'On the axiomatic theory of spectrum II', Studia Math. 119(2) (1996), 129-147.

[17] A. Pietsch, Operator Ideals (North Holland, Amsterdam-New York-Oxford, 1980).

[18] V. Rakočevič, 'Semi Browder operators and perturbations', Studia Math. 122(2) (1997), 131-137.

[19] V. Rakočevič, 'Generalized spectrum and commuting compact perturbations', Proc. Edinb. Math. Soc. 36(2) (1993), 197-209.

[20] J. Zemánek, 'The stability radius of a semi-Fredholm operator', Integral Equations Operator Theory 8(1) (1985), 137-144.

[21] S. Č. Živković-Zlatanović, D. S. Djordjević and R. E. Harte, 'Left-right Browder and left-right Fredholm operators', Integral Equations Operator Theory 69(3) (2011), 347-363.

QINGPING ZENG, School of Mathematics and Computer Science, Fujian Normal University, Fuzhou 350007, PR China

e-mail: zqpping2003@163.com 
HUAIJIE ZHONG, School of Mathematics and Computer Science, Fujian Normal University, Fuzhou 350007, PR China

ZHENYING WU, Fuzhou Strait Vocation Technological College, Fuzhou 350014, PR China 